\title{
Evaluación de competencias genéricas en estudiantes de Educación Física: una experiencia en contextos no formales
}

\author{
Assessment of generic competences in pre-service physical education teachers: An experience in non- \\ formal context \\ Rodrigo Ojeda, Bastian Carter-Thuillier, Mauricio Cresp, Sergio Sanhueza, Claudia Machuca \\ Universidad Católica de Temuco (Chile)
}

\begin{abstract}
Resumen. En el campo de la Educación Física existe la necesidad de conocer con mayor profundidad los procesos formativos que se desarrollan fuera de las instituciones educativas formales. El objetivo del presente artículo es describir la experiencia de un prácticum en contextos educativos no formales donde participan estudiantes de Educación Física, con el fin de identificar y evaluar las competencias genéricas adquiridas durante el proceso. La experiencia se desarrolla en una asignatura asociada a la carrera de Educación Física de una universidad chilena, y que tiene por objeto el desarrollo de un prácticum durante cinco meses en contextos educativos no formales, tales como: (a) centros vinculados al deporte formativo y de alto rendimiento; (b) centros vinculados a la administración y gestión deportiva; (c) centros vinculados a la actividad física y salud comunitaria; (d) gimnasios y centros de actividad física vinculados al campo del fitness. Participan de la experiencia 32 estudiantes y 18 centros representados por sus respectivos encargados. Para la recolección de datos se aplican dos cuestionarios ad-hoc, uno dirigido a los estudiantes y otro a los representantes de los centros; ambos instrumentos tienen por objeto identificar y evaluar las competencias que los alumnos evidencian durante el proceso. Los resultados muestran que según la percepción de los centros de práctica, las competencias más desarrolladas por los estudiantes durante el proceso son: (a) respeto a la diversidad; (b) adaptación al contexto; (c) trabajo colaborativo. Mientras que según la percepción del alumnado son: (a) ampliación de conocimientos; (b) respeto; (c) responsabilidad.
\end{abstract}

Palabras clave. Formación docente, Educación Física, Educación no formal, Competencias genéricas

\begin{abstract}
The Physical Education field needs to know more in depth the formative processes that take place outside formal educational institutions. The aim of this paper is to describe the experience of a practicum in non-formal educational contexts, where students of Physical Education participate, in order to identify and assess the generic competences acquired during the process. The experience is developed in a subject associated to the Physical Education Bachelor of a Chilean university and aims to develop a practicum during five months in non-formal educational contexts, such as: (a) centers linked to formative and high perfomance sports; (b) centers linked to sports administration and management; (c) centers linked to physical activity and community health; (d) gyms and physical activity centers linked to fitness. 32 students and 18 centers represented by their respective managers participate in the experience. For data collection, two ad-hoc questionnaires are applied, one addressed to students, and the other to the representatives of the centers; both instruments aim to identify and assess the competences that students show during the process. The results show that according to the perception of the practicum centers, the most developed competences by the students during the process are: (a) respect for diversity; (b) adaptation to the context; (c) collaborative work. While according to the perception of the students are: (a) expansion of knowledge; (b) respect; (c) responsibility.
\end{abstract}

Key words. Preservice Teacher Education, Physical Education, Practicums, Nonformal Education, Generic Competences.

\section{Introducción}

En el año 2006, el Instituto Internacional para la Educación Superior en América Latina y el Caribe (IESALC), publicó un informe titulado «La metamorfosis de la Educación Superior» (IESALC, 2006). En dicho documento, se muestran las principales transformaciones y desafíos de la Educación Superior en la región, destacando entre ellos: la necesidad de analizar introspectivamente los procesos formativos que se desarrollan en las universidades (Cresp, Molina \& Fernandes, 2017).

En función de lo anterior, las universidades de América Latina y el Caribe, incluido Chile, debieron examinar los modelos de formación profesional que utilizaban (Universidad de Deusto, 2007). Como resultado de dicho proceso, quedó en evidencia la necesidad de avanzar hacia la instalación de un currículo por competencias en la mayoría de las casas de estudios del país (Aguado, González, Antúnez \& de Dios, 2017; Muñoz, Sobrino, Benítez \& Coronado, 2017).

Este modelo curricular implica, una formación centrada

Fecha recepción: 10-09-18. Fecha de aceptación: 02-02-19

Bastian Carter-Thuillier

bastiancarter@gmail.com en el desarrollo de competencias profesionales integradas (Huerta, Pérez \& Carrillo, 2005; Rodríguez, Del Valle \& De la Vega, 2017). Es decir, una preparación que supere el mero aprendizaje teórico tradicional y se enfoque en el desarrollo de un conjunto de cualidades que permitan a los futuros profesionales desempeñarse exitosamente ante a las necesidades de la sociedad (Cabezas, Serrate \& Casillas, 2017; Morita-Alexander, García-Ramírez \& Escudero-Nahón, 2016). En resumen, la formación bajo este modelo debería centrarse en que los estudiantes adquieran conocimientos, habilidades y aptitudes que respondan a las exigencias reales de su campo ocupacional (Egido \& López, 2016; Gutiérrez-Díaz del Campo, García-López, Pastor-Vicedo, Romo-Pérez, Eirín-Nemiña \& Fernández-Bustos, 2016; López, Benedito \& León, 2016).

En ese sentido, diversos autores (Aguado et al., 2017; Coll, 2007; López et al., 2016) han planteado precisamente que la formación universitaria debe centrarse en el desarrollo de competencias profesionales. Es decir, que el alumnado enfrente un proceso formativo donde necesariamente integre y movilice distintos tipos de aprendizajes; afrontando situaciones y escenarios que tengan la mayor semejanza posible a su quehacer profesional futuro (Delicado, Trujillo \& García, 2018; Morita-Alexander et al., 2016). 
Ello ha significado para las universidades, una reflexión permanente sobre la formación de los estudiantes, debiendo prestar una mayor atención a las competencias personales y la interacción con la práctica (Lundvall \& Lorenz, 2010; Stiglitz \& Greenwald, 2016); además de una revisión permanente en torno al diseño, desarrollo y evaluación curricular de la docencia universitaria (López et al., 2016).

Como queda en evidencia, el modelo por competencias fomenta una formación con un alto grado de especificidad. Sin embargo, diversos autores (Delors, 1996; Ngang, Yunus \& Hashim, 2015; Pachauri \& Yadav, 2013; Villarroel \& Bruna, 2014) plantean que también es trascendental la incorporación de competencias procedimentales y actitudinales, relacionadas con el ser y el convivir. Por tanto, el desempeño profesional en una sociedad globalizada y del conocimiento, exige además de las competencias específicas de una determinada profesión, competencias genéricas o transversales. Estas últimas se expresan en diferentes desempeños, tales como: la capacidad de gestionar de forma autónoma y permanente el conocimiento, de investigar, de trabajar en equipos, de comunicarse en un segundo idioma y de aprender a lo largo de la vida (Gonzalez \& Gonzalez 2008; Le Boterf, 2001).

Lo antes descrito es válido para todos los campos ocupacionales, incluyendo la formación inicial del profesorado, proceso donde también resulta trascendental la adquisición y desarrollo de competencias específicas y genéricas, por sobre la utilización de enfoques tradicionales de enseñanza centrados sólo en el conocimiento (Bolívar, 2008; Villarroel \& Bruna, 2014; Rodríguez \& Molina, 2018).

Respecto al campo específico de la Educación Física, diferentes autores (Romero, 2004; Poblete-Valderrama, Linzmayer, Matus, Garrido \& Flores, 2017) han planteado que resulta trascendental incorporar en la formación inicial del profesorado instancias que permitan dotar de las competencias necesarias a los futuros maestros, con el fin de que puedan garantizar en el futuro, calidad y transcendencia educativa. Sin embargo, por la naturaleza y particularidades de la disciplina, la formación del profesorado de Educación Física no tan sólo debe responder a las exigencias del sistema educativo formal (UNESCO, 2015), sino que también, a otros escenarios que en términos históricos y sociales se encuentran vinculados con los profesionales del área: fitness, entrenamiento deportivo, preparación física, salud escolar (Mayorga-Vega, 2016), gestión deportiva, atención primaria de salud (Pastor-Vicedo, Gil-Madrona, Prieto-Ayuso \& González-Vilora, 2015) y centros comunitarios. En Chile el Ministerio de Educación (MINEDUC) reconoce todos estos escenarios, bajo la categoría de «contextos educativos no formales» (Álvarez, 2011; MINEDUC, 2013), siendo cada uno de ellos un posible espacio laboral para los especialistas en Educación Física.

El amplio volumen de escenarios disponibles para los profesionales del área, ha significado el surgimiento de nuevos desafíos (Zapatero, Campos \& González, 2018), y una mayor complejidad en los procesos formativos, que han derivado en cambios profundos respecto a cómo se entienden los procesos de enseñanza y aprendizaje en la disciplina (Carter-Thuillier, 2015). Lo anterior ha permeado todos los procesos didácticos e innovaciones que puedan desarrollarse en el área, incidiendo particularmente en las metodologías y estrategias de evaluación del aprendizaje (López-Pastor, 2008), por las importantes repercusiones que tiene este último aspecto en los procesos de enseñanza (Cresp et al., 2017).

El modelo por competencias implica entonces, una formación para el profesorado de Educación Física que se apegue de la forma más realista posible a la naturaleza de sus futuras funciones (Zapatero et al., 2018), lo que exige entonces, enfoques e instancias de evaluación que también deben responder a dicha lógica. En ese sentido, diferentes autores (Villarroel \& Bruna, 2014; Wiggins, 2011), sugieren el uso de una «evaluación auténtica» para la valoración de los aprendizajes y desempeños asociados a competencias específicas y genéricas. Con ello se hace referencia, a que las técnicas, instrumentos y actividades de evaluación estén centradas en situaciones, actividades y contenidos que permitan evaluar competencias en situaciones reales; es decir, que se asemejen al contexto profesional que el sujeto debe enfrentar en el futuro, evitando las situaciones puntuales y artificiales de evaluación (López, 2013)

Realizar procesos de evaluación auténtica implicaría entonces, el uso de una metodología centrada en tareas que posean estrecho vínculo con el contexto real. En ellas los estudiantes deben utilizar su conocimiento y habilidades, dando cuenta de desempeños creativos y efectivos (Fuentes \& López, 2017; Gulikers, Bastiaens \& Kirschner, 2004; López, 2013; Villarroel \& Bruna, 2014; Wiggins, 2011). Para el campo de la Educación Física, ello significa ofrecer a los futuros profesores experiencias de aprendizaje en contexto real, ya sea en el sistema educativo formal, como en contextos no formales (Martínez, Santos \& Castejón, 2017); esto favorecería su futura empleabilidad y desarrollo personal (Brown, 2015).

Un espacio coherente para la realización de evaluación auténtica serían los «prácticum» en contexto real (Blasco, Vega, Mengual, Avalos \& Zarco, 2009). Es decir, cuando el profesorado en formación asiste a centros escolares u otros contextos de educación no formal, para realizar temporalmente labores docentes, como parte su proceso de formación universitaria. Lo que permite la evaluación de desempeños específicos bajo el contexto y clima real de la profesión (Serrate, Casillas \& González, 2015; Tejada \& Ruiz, 2013; Villarroel \& Bruna, 2014; Zabalza, 2011).

En virtud de todos los antecedentes previamente expuestos, el presente artículo tiene por objetivo describir la experiencia de un prácticum en contextos educativos no formales, donde participan estudiantes de Educación Física, con el fin de identificar y evaluar las competencias genéricas adquiridas durante el proceso.

\section{Metodología}

\section{Contexto y participantes}

La experiencia se desarrolló en una universidad chilena de carácter privado, específicamente en el contexto de una asignatura llamada «Educación Física en Contextos no Formales», correspondiente al cuarto año de formación. Esta última tiene por objeto que los estudiantes puedan profundizar y valorar el rol del profesor de Educación Física en contextos de educación no formal, desarrollando 
competencias genéricas y específicas, como también la capacidad de movilizar distintos saberes disciplinarios desarrollados durante los años anteriores; los cuales están asociados a los distintos saberes competenciales: (a) saber; (b) saber hacer; (c) saber ser; (d) saber convivir.

Dentro de dicha asignatura se desarrolla un prácticum que dura cinco meses, donde cada alumno asiste cuatro horas semanales a un centro vinculado a Educación Física no formal. Durante dicho proceso, el estudiante debe ser capaz de reflexionar sobre su propia práctica, reconociendo fortalezas y áreas de mejora en su desempeño como profesor de Educación Física. Para esto, los alumnos deben diseñar e implementar una propuesta de mejoramiento personal, basada en un auto-diagnóstico que ellos mismos realizan.

Participaron de la experiencia 32 estudiantes y 18 instituciones donde el alumnado realizó su prácticum.

Los centros de práctica se clasifican en cuatro grandes categorías: (a) deporte y alto rendimiento: clubes deportivos, escuelas de iniciación deportiva, centros de alto rendimiento; (b) administración y gestión deportiva: coordinaciones de deporte de municipios, coordinaciones de deporte en universidades, Instituto Nacional del Deporte; (c) actividad física y salud comunitaria: hogares de ancianos, hogares de menores, centros de salud comunitaria, centros de tratamiento del cáncer infantil; (d) fitness: gimnasios y centros de actividad física recreativa.

\section{Técnicas e instrumentos}

Con el fin de identificar y evaluar las competencias genéricas adquiridas por los estudiantes a lo largo del prácticum se utilizaron dos instrumentos: (a) pauta de evaluación respondida por un representante del centro de prácticum: centrada en evaluar el desempeño de los estudiantes durante su práctica en el contexto real; (b) pauta de autoevaluación del alumnado: el estudiante evalúa su desempeño durante su estancia en el centro de práctica. En ambos casos se trató de instrumentos ad hoc, construidos exclusivamente para la experiencia. En su diseño participaron los profesores de la asignatura y la Dirección General de Docencia de la Universidad.

Asimismo previo a su aplicación, dichos instrumentos fueron sometidos a un proceso de pilotaje, durante los dos cursos anteriores (2016 y 2017), con el objetivo verificar su aplicabilidad y comprensión en cada uno de sus ítems. Ambos instrumentos fueron también revisados y validados por juicio de tres expertos, quienes verificaron su pertinencia, consistencia y validez teórica.

Ambos instrumentos están centrados en analizar, a través de la percepción de los actores, si los estudiantes evidencian las competencias que se exhiben en la tabla 1. Estas competencias corresponden a las delimitadas por la Dirección General de Docencia de la institución, para los estudiantes que cursan esta asignatura de prácticum.

(a) Pauta de evaluación respondida por el centro de práctica

Cada centro de práctica debió evaluar las competencias que el alumnado desarrolló durante el proceso, a través de un instrumento con respuestas en escalamiento tipo Likert.

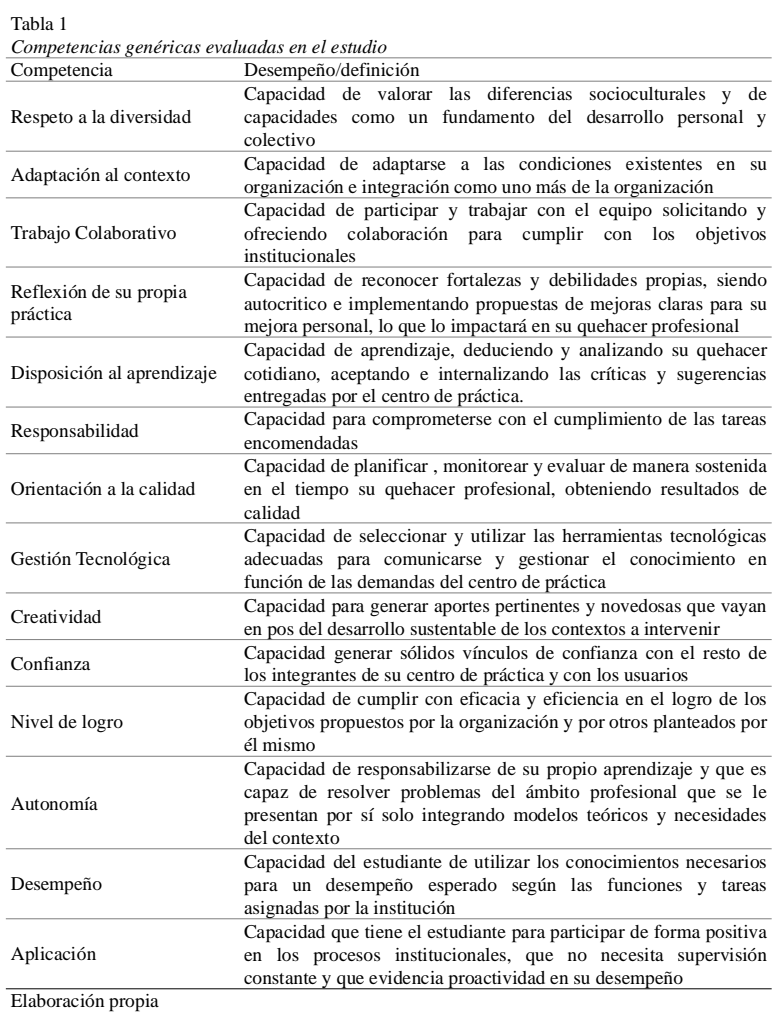

La finalidad de este último, fue recoger las valoraciones que otorgaba el profesor o tutor del centro al desempeño del alumno. El instrumento contaba de 15 preguntas (ver figura 1) con respuestas en cuatro niveles: $1=$ Deficiente; $2=$ Regular; 3= Bueno; 4= Muy Bueno.

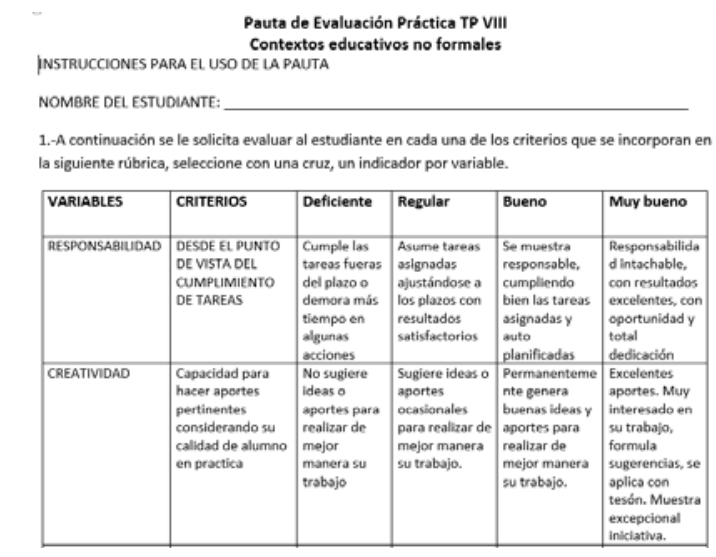

Figura 1. Ejemplo de pauta de evaluación del centro de prácticum

\section{(b) Pauta de autoevaluación del alumnado}

Asimismo, se solicitó a los estudiantes que respondieran un instrumento centrado en analizar las competencias que desarrollaron durante el prácticum. Ello con la finalidad de conocer la percepción del propio alumnado respecto a su desempeño en el proceso. El instrumento contaba de 12 preguntas (ver figura 2) con respuestas en una escala tipo Likert, de cinco niveles, en donde 1= Nunca; 2= casi nunca; 3=A veces; $4=$ Casi siempre y $5=$ Siempre.

\section{Procedimiento}

La experiencia se llevó a cabo el segundo semestre del año académico 2017. Es importante señalar que en Chile el calendario académico universitario se desarrolla desde marzo 


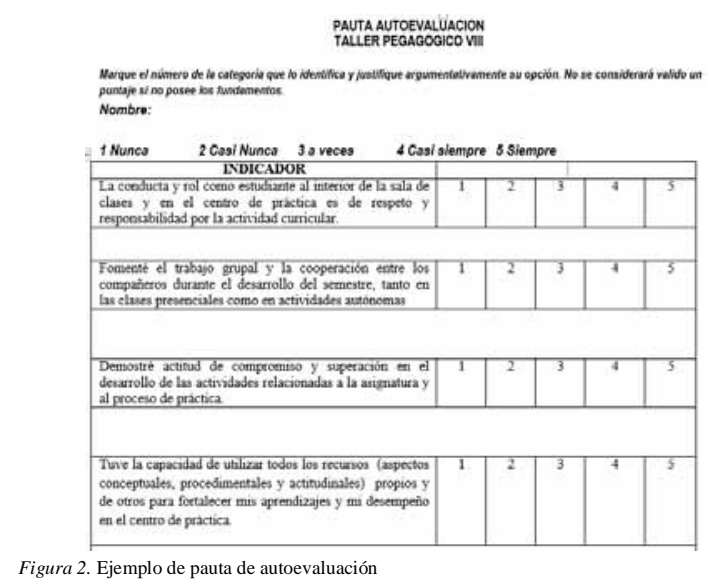

a diciembre, por lo que el segundo semestre se extiende desde agosto hasta diciembre.

Al finalizar el semestre, el profesional del centro debió completar la pauta de evaluación del prácticum y los estudiantes debieron rellenar la pauta de autoevaluación.

Los participantes fueron previamente informados de que se realizaría un proceso investigativo en torno a la experiencia en que iban a tomar parte, otorgando su consentimiento por escrito y estipulando la confidencialidad de identidad.

\section{Análisis de Datos}

En lo referido a los instrumentos con respuestas en escalamiento tipo Likert, se utilizó estadística descriptiva por variable, obteniendo la desviación típica y media aritmética de las valoraciones del alumnado utilizando el programa estadístico SPSS 24. Con ello se pretende de identificar y evaluar las competencias genéricas adquiridas durante el proceso.

\section{Resultados}

Se analizó toda la información obtenida durante la recolección de datos, a partir de los dos instrumentos aplicados.

En la tabla 2 se puede observar que según los Centros de práctica las competencias genéricas más desarrolladas por los estudiantes durante el desarrollo del prácticum son (según escala tipo Likert): (a) respeto a la diversidad, con una media de 3,87; (b) adaptación al contexto con una media de 3,80; (c) trabajo colaborativo con una media de 3,77. Las competencias que tienen una menor media son: (a) aplicación con una media de 3,30; (b) desempeño, con una media de 3,47; (c) autonomía, con una media de 3,50.

\begin{tabular}{lccc}
$\begin{array}{l}\text { Tabla } 2 \\
\text { Resultados por Media y DS según Centros de práctica }\end{array}$ & & & \\
\hline Criterio & N & Media & DS \\
\hline Respeto a la diversidad & 32 & 3,87 & 0,434 \\
\hline Adaptación al contexto & 32 & 3,80 & 0,551 \\
\hline Trabajo colaborativo & 32 & 3,77 & 0,679 \\
\hline Reflexión de su propia practica & 32 & 3,70 & 0,651 \\
\hline Disposición al aprendizaje & 32 & 3,67 & 0,661 \\
\hline Responsabilidad & 32 & 3,60 & 0,675 \\
\hline Orientación a la calidad & 32 & 3,60 & 0,621 \\
\hline Gestión tecnológica & 32 & 3,60 & 0,724 \\
\hline Aspectos administrativos & 32 & 3,53 & 0,681 \\
\hline Creatividad & 32 & 3,50 & 0,682 \\
\hline Confianza & 32 & 3,50 & 0,777 \\
\hline Nivel de logro & 32 & 3,50 & 0,682 \\
\hline Autonomía & 32 & 3,50 & 0,630 \\
\hline Desempeño & 32 & 3,47 & 0,730 \\
\hline Aplicación & 32 & 3,30 & 0,837 \\
\hline Elaboración propia & & &
\end{tabular}

En la tabla 3 se observa que según los estudiantes, los indicadores más desarrollados y valorados son: (a) ampliación de conocimientos, con una media de 4,87; (b) respeto y responsabilidad, con una media de 4,87. Las competencias que tienen una menor media son: (a) usos de espacios de retroalimentación, con una media de 4,20; (b) máximo esfuerzo, con una media de 4,27.

\begin{tabular}{lccc}
$\begin{array}{l}\text { Tabla } 3 \\
\text { Resultados por Media y DS según Autoevaluación Estudiantes }\end{array}$ & & \\
\hline Criterio & N & Media & DS \\
\hline Ampliación de conocimientos & 32 & 4,87 & 0,346 \\
\hline Respeto y responsabilidad & 32 & 4,87 & 0,434 \\
\hline Compromiso y superación & 32 & 4,73 & 0,521 \\
\hline Aprendizaje autónomo & 32 & 4,67 & 0,547 \\
\hline Fomento trabajo grupal & 32 & 4,67 & 0,547 \\
\hline Proactividad & 32 & 4,60 & 0,563 \\
\hline Responsable entrega trabajos y tareas asignadas & 32 & 4,50 & 0,900 \\
\hline Preguntas e intervenciones & 32 & 4,50 & 0,682 \\
\hline Utilización recursos & 32 & 4,50 & 0,509 \\
\hline Diálogo y discusión & 32 & 4,47 & 0,629 \\
\hline Máximo esfuerzo & 32 & 4,27 & 0,740 \\
\hline Uso espacios de retroalimentación & 32 & 4,20 & 0,961 \\
\hline Elaboración propia & & &
\end{tabular}

Los resultados antes expresados, muestran que existen similitudes entre los estudiantes y los representantes de los centros de práctica. En términos concretos, ambos actores consideran que la experiencia desarrollada colabora a la adquisición de elementos actitudinales que facilitan un mejor desempeño profesional. Aunque también se pueden observar diferencias, puesto que los estudiantes manifiestan que la experiencia fortalece la adquisición y aplicación de elementos conceptuales, aspecto no considerado por los centros de práctica.

\section{Discusión}

A continuación se desarrolla la discusión de los resultados a través de seis apartados temáticos: (a) importancia de las competencias actitudinales; (b) adaptación al contexto y compromiso de los estudiantes; (c) trabajo colaborativo y respeto a la diversidad; (d) ampliación de conocimientos en contextos reales; (e) autonomía y desempeño profesional; (f) relevancia de procesos de retroalimentación efectiva.

\section{Importancia de las competencias actitudinales}

Los resultados muestran que tanto estudiantes, como los representantes de los centros identifican un mayor desarrollo de las competencias actitudinales «respeto a la diversidad» y «adaptación al contexto» durante el prácticum, por sobre las de carácter procedimental «aplicación» y «desempeño».

Lo anterior, concuerda con lo planteado por estudios anteriores (Cabezas et al., 2017; López \& Fernández, 2010; Serrate et al., 2015), donde también se otorga una alta valoración a la adquisición de competencias genéricasactitudinales durante experiencias en contextos educativos no formales.

Lo recién expresado, releva la importancia de generar experiencias de aprendizaje relacionadas con la formación transversal, incorporando las competencias actitudinales como elemento fundamental en el proceso formativo de los futuros profesores (Barrera \& Hinojosa, 2017; López \& Fernández, 2010; Mendoza \& Covarrubias, 2014). Esto adquiere mayor importancia al revisar los resultados obtenidos, donde los encargados de los centros de práctica valoran positivamente estas competencias, lo que concuerda 
con otras investigaciones que plantean que el desarrollo de estas no sólo complementan la formación del estudiante, sino que mejoran su valoración en el mundo del trabajo (Serrate et al., 2015; Tejada \& Ruiz, 2013; Villarroel \& Bruna, 2014; Zabalza, 2011).

Respecto a lo anterior, Gore (2013) manifiesta que las competencias actitudinales son las más valoradas en el mundo laboral, por tanto, los estudiantes y futuros profesionales que cultiven este tipo de competencias tendrán una ventaja comparativa sobre su pares (Hernández, Quezada, \& Venegas, 2016; Higgins, 2013).

Adaptación al contexto y compromiso de los estudiantes

Una de las competencias más valoradas por los centros de práctica es la adaptación al contexto (3,80 \pm 0,551), entendida como la capacidad para hacer ajustes sobre la marcha e incorporarlos cuando las condiciones internas o externas cambian (Barrera \& Hinojosa, 2017; Serrate et al., 2015). Según Vallejos (2011) esta competencia suele ser un rasgo común de los trabajadores satisfechos, productivos y comprometidos con la organización. Ello corrobora lo planteado por la literatura ( Cabezas et al., 2017; González \& González, 2008; Muñoz, Medina \& Guillén, 2016; SánchezTarazaga, 2013), donde se señala que los prácticum permiten a los estudiantes universitarios fortalecer sus competencias actitudinales y procedimentales.

Lo anterior, se complementa con los resultados de los estudiantes de esta experiencia, quienes valoran positivamente las competencias de respeto y responsabilidad como aspectos relevantes para desempeñarse óptimamente en su centro de práctica. Esto último coincide con lo planteado por Mendoza y Cobarrubias (2014), quienes en una experiencia anterior, también concluyen que para los estudiantes resulta prioritario el trabajo que realizan en el centro de práctica, posicionándolo por sobre los requerimientos académicos de la propia universidad.

Esto último, encontraría explicación en el hecho de que la experiencia vivida en el centro de práctica genera mayores desafíos metodológicos y motivacionales. Por lo tanto, ello genera que el alumnado se comprometa profundamente con sus obligaciones, tanto en el centro, como en el resto de sus deberes formativos de la universidad (Mendoza \& Covarrubias, 2014).

\section{Trabajo colaborativo y respeto a la diversidad}

El trabajo colaborativo y el fomento de trabajo grupal, se encuentran dentro del primer tercio de las competencias más valoradas por los encargados de los centros de práctica (3,77 $\pm 0,679)$ y los estudiantes $(4,67 \pm 0,547)$.

Esto se condice con investigaciones anteriores, que destacan al trabajo colaborativo como una de las competencias más valoradas por los empleadores, siendo identificada como fundamental para una participación productiva en la sociedad del siglo XXI (Baltar, Carrasco, Jensen, Villegas \& Tapia, 2012; Diaz, 2010; Scott, 2015). Dicha competencia significa la integración y colaboración activa en la consecución de objetivos comunes con otras personas, áreas y organizaciones, permitiendo soluciones interdisciplinarias a los desafíos laborales (Hernández et al., 2016; Rodríguez et al., 2017).
Asimismo la literatura actual (Castro, 2018; Durán \& Giné, 2017; Navarro, López \& Hernández, 2017) plantea que el trabajo colaborativo se asocia directamente con el respeto hacia la diversidad, puesto que permite la construcción de relaciones interpersonales sustentadas en el diálogo igualitario y la interdependencia positiva. Por este motivo, cuando el grupo es amplio y se diversifica, es importante que todo el mundo conozca el trabajo de los demás y que existan momentos de intercambio que lo fomenten (López et al., 2005).

\section{Ampliación de conocimientos en contextos reales}

Los estudiantes afirman que durante el prácticum adquieren y profundizan nuevas competencias profesionales. Ello se posiciona como una de las fortalezas de la experiencia en cuestión.

Respecto a lo antes expresado, se puede señalar que estudios anteriores (Delicado et al., 2018; Gutiérrez-Díaz del Campo et al. 2016; Raposo-Rivas \& Martínez-Figueira, 2013; Tejada \& Ruiz, 2013) muestran que la adquisición de competencias es significativamente mayor, cuando el proceso se desarrolla bajo condiciones que se asemejan a la realidad profesional. Es decir, situaciones de aprendizaje auténticas, centradas en el protagonismo del estudiante.

En este sentido, Delicado et al. (2018), Latorre y Blanco (2011), además de Tejada y Ruiz (2013), plantean que el desarrollo de un prácticum contextualizado y ligado a la teoría, mediante una postura crítica y reflexiva a la vez, propicia la ampliación de conocimientos, el desarrollo de la autonomía, la responsabilidad y un análisis continuo respecto a los problemas que plantea el prácticum; por lo tanto se convierte en un espacio privilegiado de aprendizaje profesional.

\section{Autonomía y desempeño profesional}

Los estudiantes perciben que durante el prácticum adquieren un alto grado de autonomía y desempeño profesional. Sin embargo, los representantes de los centros no coinciden con esta percepción, evaluando dicha competencia como una de las más débiles en el alumnado.

Lo antes descrito coincide con estudios anteriores (Montecinos, Walker \& Cortez, 2015; Serrate et al., 2015), donde también existió disparidad entre la percepción de los estudiantes y sus respectivos centros de práctica. Esto se debe habitualmente a las escasas instancias de retroalimentación formal que los estudiantes reciben por parte de la universidad y sus centros de práctica durante el proceso, lo que genera en los estudiantes una percepción distinta (Gallardo \& Carter-Thuillier, 2016; Insuasty \& Zambrano, 2011; Montecinos, Barrios \& Tapia, 2011).

Esta percepción del alumnado podría estar asociada al elevado volumen de horas que cada alumno destina semanalmente al proceso de prácticum, además de su compromiso y proactividad con el centro. De hecho, como ha quedado en evidencia, los estudiantes de forma autónoma adquieren nuevas competencias para contestar a las demandas del centro.

En ese sentido, varios autores (Zimmerman \& Schunk, 2001; López et al., 2016) plantean que las experiencias en contexto real facilitan el desarrollo de la autonomía y autorregulación por parte de los estudiantes, quienes deben movilizar distintas estrategias de aprendizaje y asumir un 
importante grado de compromiso para el logro de las metas. Asimismo, Pérez, Valenzuela, Diaz, González y Núñez (2011) expresan que en este tipo de experiencias prácticas, los estudiantes tienden a utilizar estrategias profundas de aprendizaje, propendiendo al desarrollo de habilidades superiores del pensamiento, lo que impactará en la adquisición de aprendizajes significativos y perdurables en el tiempo.

En este sentido, diferentes autores (Gálvez \& Martín, 2016; López et al., 2016) manifiestan que una de las mayores dificultades que enfrentan actualmente los estudiantes universitarios, es el desarrollo de competencias de aplicación y desempeño. Ello convierte a las experiencias de prácticum, como la presente, en una opción real para contestar dicho problema (Hernández del Campo et al., 2016; Tejada \& Ruiz, 2013).

\section{Relevancia de los procesos de retroalimentación efectiva}

$\mathrm{Al}$ analizar los resultados de la experiencia, se aprecia que estudiantes y encargados de centros de práctica tienen una percepción dispar respecto al logro de algunas competencias. Estas discrepancias pueden asociarse a las relaciones particulares que cada estudiante construye con el profesor del centro de práctica; lo que condiciona los procesos de retroalimentación y aprendizaje. Asimismo, en cada centro existen profesionales con diferente nivel de experiencia o conocimiento respecto a las competencias que se espera desarrollen los estudiantes en su prácticum (Insuasty \& Zambrano, 2011; Zaragoza, Luis-Pascual \& Manrique, 2009). Esta situación se complejiza, ya que, en algunos centros de práctica, este rol de supervisor lo realizan profesionales de distintas áreas disciplinares y no necesariamente profesores de Educación Física (Egido \& López, 2016).

En este sentido Prieto (2007) plantea que una de las características que debe tener un observador para lograr una retroalimentación de calidad y desarrolle la autoeficacia, es ser considerado competente por parte de quien recibe la retroalimentación. Independiente de sus características personales, el observador deberá presentarse como un experto cercano, que pueda reconocer los progresos de los estudiantes, así como dar a conocer múltiples soluciones a una problemática determinada, además de entregar de estrategias u orientaciones claras para la solución de problemáticas, entre otros.

\section{Conclusiones}

A partir de los resultados de la experiencia, se puede señalar que las competencias con mayor valoración por parte de los centros de práctica son: (a) respeto a la diversidad; (b) adaptación al contexto; (c) trabajo colaborativo. Es decir, los centros consideran que estas son las competencias más relevantes que adquirieron los alumnos durante el período de prácticum. Por otra parte, las competencias que tienen una menor valoración son: (a) aplicación; (b) desempeño; (c) autonomía.

Según los estudiantes, los indicadores más desarrollados y valorados son: (a) ampliación de conocimientos; (b) respeto; (c) responsabilidad. Las competencias que tienen menor impacto o efecto son: (a) usos de espacios de retroalimentación y;(b) máximo esfuerzo.

La experiencia expuesta en el presente documento, coincide con la literatura existente. Puesto que describe un proceso de aprendizaje relacionado directamente con el contexto y las competencias del quehacer profesional, por lo tanto se trata de una situación de aprendizaje auténtica. En ese sentido, queda en evidencia la valoración que se otorga a las competencias actitudinales como elemento fundamental en el proceso formativo de los futuros profesores.

A partir de los resultados, parece indispensable considerar en la formación del profesorado de Educación Física, la incorporación de actividades curriculares que se vinculen directamente al mundo laboral, incluyendo los contextos de educación no formal. Asimismo, queda en evidencia que el uso de estrategias activas-participativas en contexto real, permite al alumnado la adquisición y profundización de nuevas competencias, además de la integración, aplicación y conexión de las ya adquiridas previamente en el aula; fomentando con ello aprendizajes significativos y perdurables en el tiempo.

Sin embargo, parece también necesario establecer mecanismos de retroalimentación sistemática por parte de las universidades, que permitan a los estudiantes generar procesos de reflexión y autorregulación frente a los diferentes desafíos que surjan en el desarrollo de su prácticum.

La experiencia reflejada en el presente artículo, puede ser de interés para el profesorado de Educación Física, así como para los profesionales vinculados con la formación por competencias y los contextos de educación no formal. Asimismo, los resultados de esta experiencia pueden resultar de utilidad para la mejora de los procesos vinculados a la formación inicial del profesorado.

Los hallazgos expuestos en el presente manuscrito, corresponden a los resultados de una experiencia desarrollada en una realidad educativa particular. Por lo tanto, todas las posibles inferencias que se puedan hacer a partir de los datos recolectados, son válidos y aplicables para dicho contexto. En virtud de lo anterior, es recomendable desarrollar nuevas experiencias e investigaciones en otros escenarios, que permitan conocer con mayor precisión los alcances formativos que pueden tener las experiencias en contextos no formales, sobre la formación del profesorado de Educación Física.

A modo de prospectiva, sería valioso realizar estudios que profundicen al menos dos ámbitos: (a) determinar el impacto que poseen los prácticum en contextos formales, en la formación de profesores vinculados al campo de la Educación Física; (b) analizar si los profesores en formación extrapolan al contexto formal, aquellas competencias adquiridas en los centros de práctica de educación no formal.

\section{Referencias}

Aguado, D., González, A., Antúnez, M., \& de Dios, T. (2017). Evaluación de Competencias transversales en universitarios. Propiedades psicométricas iniciales del cuestionario de competencias transversales. Revista iberoamericana sobre calidad, eficacia y cambio en educación, 15(2), 129-152. 
Alvarez, C. (2011). Rol del profesor de educación física en la atención primaria de salud pública y su potencial injerencia en las metas sanitarias desde la promoción, prevención y rehabilitación de la salud. Lecturas de educación física y deportes, 16(156).

Baltar, J., Carrasco, C., Jensen, D., Villegas, C., \& Tapia, N. (2012). El trabajo interdisciplinario entre psicólogos y profesores: Estudio cualitativo sobre los significados de asesores técnicos en educación respecto de su experiencia como equipo de trabajo. Revista de psicología, 21(2), 187-213.

Barrera, M., \& Hinojosa, C. (2017). Incidencia del proceso de la práctica profesional en las dimensiones de la formación docente de estudiantes de pedagogía en educación física Pensamiento educativo, 54(2), 1-15.

Blasco, J., Vega, L, Mengual, S., \& Zarco, P. (2009). Evaluación de las competencias docentes en el practicum de Educación Física. En Rapaso, M., Martínez, M., Lodoiro, L., Fernández, J., \& Pérez, A. (Ed.), X Symposium Internacional sobre Practicum y Prácticas en empresas en la formación universitaria. El Practicum más allá del empleo. Formación vs Training. 1233-1246. Poio, Pontevedra.

Bolívar, A. (2008). El discurso de las competencias en España: Educación básica y educación superior. Revista de docencia universitaria, 6(2), 1-23.

Brown, R. (2015). La evaluación auténtica: El uso de la evaluación para ayudar a los estudiantes a aprender. Revista electrónica de investigación y evaluación educativa, 212, 1-10.

Cabezas, M., Serrate, S., \& Casillas, S. (2017). Valoración de los alumnos de la adquisición de competencias generales y específicas de las prácticas externas. Factores determinantes. Revista mexicana de investigación educativa, 22(74), 685704.

Carter-Thuillier, B. (2015). Evaluación para el aprendizaje: Innovación, democracia y transformación en educación física. En M. Cresp, J. Serra-Olivares, B. Carter-Thuillier y R. García (Eds.), Evaluación para el aprendizaje: Educación Física e Innovación en procesos formativos, 24-49. Madrid, España: Lambert-EAE.

Castro, R. (2018). El desarrollo de competencias para el trabajo docente en escuelas multigrado. Revista iberoamericana para la investigación y el desarrollo educativo, 8(16), 335350.

Coll, C., (2007). Las competencias en la educación escolar: Algo más que una moda y mucho menos que un remedio. Aula de innovación educativa, 16(1), 24-39.

Cresp, M., Molina, P., \& Fernandes, J. (2017). La Educación superior en Chile: Cambios paradigmáticos de los procesos evaluativos. Del proceso tradicional de formación a los modelos por competencias. Revista dilemas contemporáneos, 5(1), 1-17.

Delicado, M., Trujillo, J., \& García, L. (2018). Valoración sobre la formación en la mención de Educación Física, por parte del alumnado de Grado en Educación Primaria. Retos, 34, 194 199.

Delors, J. (1996). La educación encierra un tesoro. Paris: Ediciones Unesco.

Díaz, J. (2010). Educación física e interdisciplinaridad: Una relación cada vez más necesaria. Revista tandem, 33, 7-21.

Durán, D., \& Giné, C. (2017). La formación del profesorado para la educación inclusiva: Un proceso de desarrollo profesional y de mejora de los centros para atender la diversidad. Revista latinoamericana de educación inclusiva. 5(2), 153-170.

Egido, I., \& López, E. (2016). Condicionantes de la conexión entre la teoría y la práctica en el Prácticum de Magisterio: Algunas evidencias a partir de TEDS-M. Estudios sobre educación, 30,217-237.

Fuentes, T., \& López, V. (2107). Evaluación auténtica, coevaluación y uso de las TIC en educación física: Un estudio de caso en secundaria. Infancia, educación y aprendizaje. 3(2), 42-46.

Gallardo, F., \& Carter-Thuillier, B. (2016). La evaluación formativa y compartida durante el prácticum en la formación inicial del profesorado: Análisis de un caso en Chile. Retos. Nuevas tendencias en Educación Física, Deporte y Recreación, 29, 258-263.

Gálvez, I., \& Martín, E. (2016). Condicionantes de la conexión entre la teoría y la práctica en el prácticum de magisterio: Algunas evidencias a partir de TEDS-M. Estudios sobre educación, 30, 217-237.

González, V., \& González, R. (2008). Competencias genéricas y formación profesional: Un análisis desde la docencia universitaria. Revista iberoamericana de educación, 47, 185210.

Gore, V. (2013). 21st Century skills and prospective job challenges. Journal of soft skills, 7(4), 7-14.

Gulikers, J., Bastiaens, T., \& Kirschner, P. (2004). A FiveDimensional framework for authentic assessment. Educational technology research and development, 52(3), 67-86.

Gutiérrez-Díaz del Campo, D., García-López, L., Pastor-Vicedo, J., Romo-Pérez, V., Eirín-Nemiña, R., \& Fernández-Bustos, J. (2016). Percepción del profesorado sobre la contribución, dificultades e importancia de la Educación Física en el enfoque por competencias. Retos, 31, 34-39.

Hernández, M., Quezada, A., \& Venegas, M. (2016). Análisis de la práctica docente en la formación inicial de profesores de religión. Educación y educadores, 19(3), 357-369.

Higgins, J. (2013). The fourth singularity and the future of jobs. World futures review, 5(1), 11-23.

Huerta, J., Pérez, I. \& Carrillo, G (2005). Referentes conceptuales para la enseñanza centrada en el aprendizaje. Revista de educación y desarrollo, 4, 34-44.

Instituto Internacional para la Educación Superior en América Latina y el Caribe (2006). Informe sobre la educación superior en América Latina y el Caribe. 2000-2005. La metamorfosis de la educación superior. Venezuela: Editorial Metrópolis C.A.

Insuasty, E., \& Zambrano, L. (2011). Caracterizacion de los procesos de retroalimentación en la práctica docente. Entornos, 24, 73-85.

Latorre, M., \& Blanco, F. (2011). El prácticum como espacio de aprendizaje profesional para docentes en formación. Revista de docencia universitaria, 9(2), 35-54.

Le Boterf, G (2001). Ingeniería de las competencias. Barcelona: Epise.

López, C., Benedito, V., \& León, M. (2016). El Enfoque de competencias en la formación universitaria y su impacto en la evaluación. La perspectiva de un grupo de profesionales expertos en pedagogía. Formación universitaria, 9(4), 1122.

López, V., Monjas, R., Barba, J., Suttil, P., González, M., García, J.,...Martín, M. (2005). Doce años de investigación-acción en educación física. La importancia de las dinámicas colaborativas en la formación permanente del profesorado. El caso del grupo de trabajo internivelar de Segovia. Lecturas de educación física y deportes, 10(90).

López, V. (2008). La importancia del trabajo colaborativo para el tratamiento de la motricidad infantil. Una propuesta integral 
a partir del aprendizaje inducido. Revista española de pedagogía, 239, 137-150.

López, V. (2013). Nuevas perspectivas sobre evaluación en educación física. Revista de educación física, 29(3), 4-13.

Lundvall, B., \& Lorenz, E. (2010). Innovación y desarrollo de competencias en la economía del aprendizaje. Implicaciones para las políticas de innovación. Innovación y aprendizaje, 44-101.

Martínez, F., Santos, M., \& Castejón, F. (2017). Percepciones de alumnado y profesorado en educación superior sobre la evaluación en formación inicial en educación física. Retos. Nuevas tendencias en Educación Física, Deporte y Recreación, 32, 76-81.

Mayorga-Vega, D., (2016). Efectos del refuerzo intermitente sobre el mantenimiento de la condición física orientada a la salud en la educación física escolar. (Tesis doctoral). Universidad de Granada, Granada.

Mendoza, M., \& Covarrubias, C. (2014). Competencias profesionales movilizadas en el prácticum: Percepciones del estudiantado del grado de maestro en educación primaria. Actualidades investigativas en educación, 14(3), 1-24.

Ministerio de Educación de la República de Chile. (2013). Estándares orientadores para carreras de pedagogía en educación física. Santiago: MINEDUC.

Montecinos, C., Barrios, C., \& Tapia, M. (2011). Relación entre estilos de supervisión durante la práctica profesional y las creencias de autoeficacia de los estudiantes de pedagogía en educación general básica. Perspectiva educacional, 50(2), 96-122.

Montecinos, C., Walker, H., \& Cortez, M. (2015). Sugerencias de docentes directivos para mejorar la formación práctica en las carreras de pedagogía: Transitando de acciones fragmentadas a una participación legítima en los colegios. Estudios pedagógicos, 41(2), 157-176.

Morita-Alexander, A., García-Ramírez, T., \& Escudero-Nahón, A. (2016). Analysis of the perception of generic competences in higher education institutions in Mexico. Revista de educación y desarrollo, 38, 69-78.

Muñoz, F., Medina, A., \& Guillén, M. (2016). Jerarquización de competencias genéricas basadas en las percepciones de docentes universitarios. Educación química, 27(2), 126-132.

Muñoz, V., Sobrino, M., Benítez, L., \& Coronado, A. (2017). Revisión sistemática sobre competencias en desarrollo sostenible en educación superior. Revista iberoamericana de educación, 73, 85-108.

Navarro, M., López, A., \& Hernández, M. (2017). El trabajo colaborativo en red impulsor del desarrollo profesional del profesorado. Revista brasileira de educação, 22(70), 651667.

Ngang, T., Yunus, H., \& Hashim, N. (2015). Soft skills integration in teaching professional training: Novice teachers' perspectives. Procedia-social and behavioral sciences, 186, 835-840.

Pachauri, D., \& Yadav, A. (2013). Importance of soft skills in teacher education programme. International journal of educational research, 41(5), 22-25.

Pastor-Vicedo, J.C., Gil-Madrona, P., Prieto-Ayuso, A., \& GonzálezVilora, S. (2015). Los contenidos de salud en el área de educación física: Análisis del currículum vigente. Retos. Nuevas tendencias en Educación Física, Deporte y Recreación, 28, 134-140.

Pérez, M., Valenzuela, M., Diaz, A., González, J., \& Núñez, J. (2011). Disposición y enfoques de aprendizaje en estudiantes universitarios de primer año. Universitas psychologica, 10(2),
441-449.

Poblete-Valderrama, F., Linzmayer, L., Matus, C., Garrido, A., \& Flores, C. (2017). Percepción de estudiantes de Pedagogía en Educación Física hacia sus. Retos, 33, 143-147.

Prieto, L. (2007). Autoeficacia del profesor universitario: Eficacia percibida y praìctica docente. Madrid: Ediciones Narcea.

Raposo-Rivas, M.; \& Martínez-Figueira, S. (2013). Mentoring student training, what is important? The new educational review. 32(2), 243-254.

Rodríguez, J., \& Molina, M. (2018). Percepción del alumnado de segundo de bachillerato sobre su formación en competencias. Revista complutense educación, 29(2), 509526.

Rodríguez, I., Del Valle, S. \& De la Vega, R. (2017). Revisión nacional e internacional de las competencias profesionales de los docentes de Educación Física. Retos, 34, 393-388.

Romero, C. (2004). Argumentos sobre la formación inicial de los docentes en educación física. Profesorado, 8(1), 1-20.

Sánchez-Tarazaga, V. (2013). Importancia de las competencias docentes según el profesorado de secundaria de la provincia de Castellón. Fòrum de Recerca, (17), 403-420.

Scott, C. (2015). El futuro del aprendizaje ¿Qué tipo de pedagogías se necesita para el siglo XXI?. Paris: Ediciones Unesco.

Serrate, S., Casillas, S., \& González, M. (2015). Percepción de los estudiantes del grado de pedagogía y educación social sobre la organización, la utilidad y formación del practicum. Enseñanza \& Teaching, 33(2), 171-190.

Stiglitz, J., \& Greenwald, B. (2016). La creación de una sociedad del aprendizaje. Madrid: Editorial la esfera de los libros.

Tejada, J., \& Ruiz, C. (2013). Significación del prácticum en la adquisición de competencias profesionales que permiten la transferencia de conocimiento a ámbitos propios de la acción docente. Profesorado. 17(3), 91-110.

United nations educational, scientific and cultural organization (2015). Educación física de calidad. Guía para los responsables políticos. París: Ediciones Unesco.

Universidad de Deusto (2007). Reflexiones y perspectivas de la educación superior en América Latina. Deusto: Publicaciones Universidad de Deusto.

Vallejos, O. (2011). Adaptación laboral: Factor clave para el rendimiento y la satisfacción en el trabajo. Cultura, educación y sociedad, 2(1), 171-176.

Villarroel, V., \& Bruna, D. (2014). Reflexiones en torno a las competencias genéricas en educación superior: Un desafío pendiente. Psicoperspectivas, 13, 23-34.

Wiggins, G (2011). Moving to modern assessments. Phi delta kappan, 92(7), 63-63.

Zabalza, M. (2011). El practicum en la formación universitaria: Estado de la cuestión. Revista de Educación, 354, 21-43.

Zapatero, J., Campos, A., \& González M. (2018). La formación inicial y permanente del profesorado de educación física para la aplicación del modelo competencial: Un estudio cualitativo. Revista complutense de educación, 29(1), 251267.

Zaragoza, J., Luis-Pascual, J., \& Manrique, J. (2009). Experiencias de innovación en docencia universitaria: Resultados de la aplicación de sistemas de evaluación formativa. Revista de docencia universitaria, 7(4), 1-33.

Zimmerman, B., \& Schunk, D. (2001). Self-regulated learning and academic achievement: Theoretical perspectives. New York:Lawrence erlbaum associates. 